\title{
Multivariate Mixed Linear Model Analysis of Longitudinal Data: An Information-Rich Statistical Technique for Analyzing Plant Disease Resistance
}

\author{
Yogasudha Veturi, Kristen Kump, Ellie Walsh, Oliver Ott, Jesse Poland, Judith M. Kolkman, \\ Peter J. Balint-Kurti, James B. Holland, and Randall J. Wisser
}

First and ninth authors: Department of Plant and Soil Sciences, University of Delaware, Newark 19716; second, fourth, and eighth authors: Department of Crop Science, North Carolina State University, Raleigh 27695; third author: Department of Plant Pathology, Ohio State University, Wooster 44691; fifth author: Hard Winter Wheat Genetics Research Unit, U.S. Department of Agriculture-Agricultural Research Service (USDA-ARS), Manhattan, KS 66506; sixth author: Department of Plant Pathology and Plant-Microbe Biology, Cornell University, Ithaca, NY 14853; seventh author: Department of Plant Pathology, North Carolina State University, Raleigh; and seventh and eighth authors: Plant Science Research Unit, USDA-ARS, Raleigh, NC 27695.

Accepted for publication 12 July 2012.

\begin{abstract}
Veturi, Y., Kump, K., Walsh, E., Ott, O., Poland, J., Kolkman, J. M., Balint-Kurti, P. J., Holland, J. B., and Wisser, R. J. 2012. Multivariate mixed linear model analysis of longitudinal data: An information-rich statistical technique for analyzing plant disease resistance. Phytopathology 102:1016-1025.

The mixed linear model (MLM) is an advanced statistical technique applicable to many fields of science. The multivariate MLM can be used to model longitudinal data, such as repeated ratings of disease resistance taken across time. In this study, using an example data set from a multienvironment trial of northern leaf blight disease on 290 maize lines with diverse levels of resistance, multivariate MLM analysis was performed

genotypic effects were highly correlated across disease ratings and followed an autoregressive pattern of correlation decay. Because longitudinal data are often converted to the univariate measure of area under the disease progress curve (AUDPC), comparisons between univariate MLM analysis of AUDPC and multivariate MLM analysis of longitudinal data were made. Univariate analysis had the advantage of simplicity and reduced computational demand, whereas multivariate analysis enabled a comprehensive perspective on disease development, providing the opportunity for unique insights into disease resistance. To aid in the application of multivariate MLM analysis of longitudinal data on disease resistance, annotated program syntax for model fitting is provided for the software ASReml.
\end{abstract} and its utility was examined. In the population and environments tested,
The mixed linear model (MLM) is a flexible modeling framework in which less-restrictive assumptions about the structure of experimental data can be made and a broad scope of inference can be achieved. Merits of the MLM for studying data on plant disease resistance have been recognized (11) and several plant pathology studies have exploited MLMs $(15,20,21,23,25,30)$. The ability to fit MLMs in semi-user-friendly software packages such as SAS (SAS Institute Inc., Cary, NC), R (R Development Core Team 2010, Vienna), and ASReml (VSN International Ltd., UK) has presented the opportunity for continued exploitation of MLMs in plant pathology studies. In this study, we present the application of the multivariate MLM to the analysis of a designed multi-environment trial in which disease resistance data were repeatedly measured across time on a relatively large population sample.

A common way to measure plant disease is to collect longitudinal data in designed experiments (i.e., repeated ratings of disease resistance taken on each experimental unit, such as rows or plots in a field) across time. For such longitudinal data, correlations between ratings are expected to exist among and within the experimental units of a trial (7). The former represents correlation

Corresponding author: R. J. Wisser; E-mail address: rjw@udel.edu

*The $\boldsymbol{e}$-Xtra logo stands for "electronic extra" and indicates that the online version contains two supplementary files.

http://dx.doi.org/10.1094/PHYTO-10-11-0268

(c) 2012 The American Phytopathological Society among the levels of the treatment and design factors in an experiment (e.g., correlation among genotypes between different ratings). Patterns of change in the correlations between ratings across time can exist for each factor, giving rise to factor-specific correlation structures including autoregressive, uniform, banded, or others (2). The latter represents correlation between timespecific residuals. These residual values can comprise multiple sources of variation that include measurement error (expected to have no pattern of correlation across time), biological fluctuations (expected to have some pattern of correlation across time), and any unaccounted-for correlation in the model (expected to have an unknown pattern of correlation across time). Fitting a multivariate MLM allows the correlative structure underlying a data set to be identified and its parameters estimated.

Ever since the longitudinal nature of disease progression was thoroughly described by Vanderplank in the 1960s (28), analysis of longitudinal data on disease resistance has been approached in different ways, including the modeling of area under the disease progress curve (AUDPC; in which the multivariate structure of the data is converted into a univariate one) (14) and modeling functional relationships between disease development and time $(4,6,16,19,20,31)$. Madden et al. (21, page 104) suggested several cases in which multivariate analysis of longitudinal data would be useful, including cases when only few ratings on disease development are available or when all of the levels of a treatment do not follow a common disease development model (i.e., cases where it is difficult or inappropriate to assume a single mathematical function with which to model the data). However, to our knowledge, 
multivariate MLM analysis of longitudinal data on disease resistance with factor-specific correlative structures has not been examined. Some key features of using multivariate MLM for analysis of longitudinal data are that it requires no assumption about the pattern of disease development across time, allows for the characterization of treatment effects in a time-specific manner, can be used to study traits collected across time that may or may not be the same (e.g., juvenile and adult plant resistance), and can allow for the estimation of missing observations based on the correlative structure of the data. Thus, this study was focused on the application of the multivariate MLM to model longitudinal disease resistance data and analysis of its correlative structure with emphasis on examining random effect terms with large numbers of treatment levels (common in plant breeding and genetics); however, the framework presented here can be readily extended to other studies in plant pathology.

Because many studies have transformed and analyzed longitudinal data as AUDPC, we considered how it compares with multivariate analysis. Statistical techniques can be evaluated in terms of (i) ease of application, (ii) appropriateness of the statistical modeling procedure given the questions and the data, (iii) power of the hypotheses tests, and (iv) informativeness of the analysis. Modeling univariate (AUDPC) data is certainly easier than modeling longitudinal data. Fitting multivariate models to longitudinal data requires the exploration, estimation, and interpretation of its underlying correlation structures. However, calculating AUDPC may not be the most appropriate approach in all cases. Because AUDPC summarizes only the mean of change across time, it is possible for treatments to significantly differ in disease development and yet exhibit nonsignificant differences in AUDPC (27). In contrast, multivariate analysis is conducted with all of the time-specific measurements and, therefore, can detect differences in disease development where AUDPC may not.

With respect to statistical power, the number and timing of disease ratings taken on each experimental unit can have an impact. Increasing the number of ratings will lead to a more accurate representation of disease progress curves as well as an increase in the power to detect differences in treatment effects. For multivariate analysis, it has been shown that increasing the number of treatment replications can lead to substantially greater increases in power than increasing the number of repeated measurements $(9,10,26)$. However, these studies have only considered situations in which treatment and residual variation across time were assumed constant, which is not expected to be the case in real-world experiments, including disease trials (it is likely that variances will change across time). Therefore, the timing of ratings may also impact power. With respect to informativeness, analysis based on AUDPC does not provide information about patterns of change across time, leading to a loss of potentially valuable information. In contrast, the multivariate MLM provides such information, including the change in means, variances, and trait heritabilities over time, which can help to identify the ways in which specific treatments affect disease progression. Furthermore, when analyzing longitudinal data as AUDPC, missing ratings can lead to biased estimates and a further reduction in informativeness and statistical power. There are circumstances when the same number of ratings cannot be taken on each experimental unit leading to moderate amounts of missing observations. The multivariate MLM accommodates such imbalance in the data and can even predict the missing information (15).

In this study, we applied multivariate MLM analysis to longitudinal data collected from trials of northern leaf blight (NLB) disease on maize. Some guidelines and computer syntax are provided for multivariate MLM analysis using ASReml, a comprehensive mixed-modeling software package now freely available to academic researchers; this is intended to help researchers use multivariate MLM analysis on their data sets. Comparisons of multivariate MLM analysis of longitudinal data and univariate analysis of AUDPC were conducted with respect to interpretations of genetic variation and disease development, and an exploratory technique to identify treatment effects producing atypical response profiles was developed. Furthermore, a simulation analysis was conducted to examine how the number and timing of disease ratings affects the estimation of heritability at different levels of between-rating correlation among treatment levels.

\section{THEORY AND APPROACHES}

Experimental materials. Genetic stocks were derived from a maize population previously subjected to recurrent mass selection for components of NLB resistance caused by the fungus Setosphaeria turcica (Luttrell) (anamorph: Exserohilum turcicum; syn. Helminthosporium turcicum). The originally selected populations have been described previously (5). Briefly, selections were initiated from a synthetic population developed from five maize inbred lines with quantitative differences in resistance to NLB: 69-1, Mo17, B37, A619, and A632. The inbred lines were reported to be recessive at the major $H t$ genes known to confer qualitative or single-gene race-specific resistance to NLB; selection was reportedly for quantitative rather than qualitative resistance. To determine whether selection for component traits of quantitative resistance (i.e., latent period and lesion length expansion rate assayed pre-flowering) could be used for population improvement, Carson (5) conducted three cycles of parallel selection for each of these two traits. Starting from the same base cycle (a synthetic population), selection was carried out separately for increasing the latent period (iLP) and decreasing the rate of lesion expansion (dLE). Carson (5) demonstrated that selection for these component traits translated into significant gains over cycles of selection in post-flowering (adult) plant resistance, measured as AUDPC.

The genetic stocks produced for this study comprised a random sample of 58 individuals from each of five different cycles: $\mathrm{C}_{0}$, $\mathrm{C}_{1}$-dLE, $\mathrm{C}_{3}$-dLE, $\mathrm{C}_{1}$-iLP, and $\mathrm{C}_{3}$-iLP. Seed of $\mathrm{C}_{2}$-dLE and $\mathrm{C}_{2}$-iLP was not available for study. The individuals sampled were selfpollinated to produce a metapopulation of 290 families. We refer to the sampled individuals as $S_{0}$ plants and their respective selfpollinated progenies as $S_{0: 1}$ families. The $S_{0}$ plants were sampled from the progeny of a single generation of random mating (a seed increase) that Carson (5) produced prior to the final evaluation of the populations' response. In addition, to serve as check lines in field trials and to provide information about the inheritance of NLB resistance (part of another study), we included a full diallel among the five known founders of the synthetic population, giving rise to a total of 315 distinct entries for trialing.

Experimental design and data collection. Field trials were conducted at two locations, Clayton, NC and Aurora, NY, for 2 years, 2007 and 2008 (environmental abbreviations: NC07, NC08, NY07, and NY08). Each trial included 320 entries; among the checks, the five founder inbred lines were present twice within each replication. The experimental design was a 20-by-16 $\alpha$ lattice with three replications at each location. In each trial, single-row plots were thinned to an approximate density of one plant per $20 \mathrm{~cm}$ and artificially inoculated with $S$. turcica. In North Carolina, sorghum grain preinoculated with a diverse mixture of $S$. turcica isolates (including race 1, race 2,3, and race $2,3, \mathrm{~N})$ was used as inoculum. In New York, both preinoculated sorghum grain and a spore suspension of the same single race 1 isolate (NY-001 from the laboratory of R. J. Nelson) were used as inoculum. Inoculations were conducted between the sixth and eight leaf stages by applying $\approx 20$ grains of the sorghum culture to the whorl; in addition, in New York, $0.5 \mathrm{ml}$ of a spore suspension of $4 \times 10^{3} \mathrm{conidia} / \mathrm{ml}$ was applied. Fields were irrigated following inoculations in North Carolina while no irrigation was applied in 
New York. One of the replications in NC07, located in a separate field from the other two replications, was discarded due to poor plant growth and disease development.

Data were collected on flowering time and adult disease resistance. Resistance was measured post-anthesis based on visual assessment of all but the end plants in each single-row plot (i.e., experimental unit). Disease leaf area was recorded as the visually averaged symptomatic leaf area per plot on a scale of 0 to $100 \%$. When measuring diseased leaf area, multiple ratings were taken on the same experimental units at $\approx 10$-day intervals, initiated at $\approx 10$ days after the average day of anthesis for the entire set of entries. In NY07, three ratings were recorded, whereas four ratings were recorded in the other three environments. In $\mathrm{NC} 07$ and NC08, a qualitatively distinguishable chlorotic lesion was observed in some plots and its occurrence in each plot was recorded on a binary scale as present or absent. Due to the differential competition of end plants within a plot, end plants were excluded from all measurements.

Statistical analysis. The diseased leaf area data were squareroot transformed to achieve normality and correct for heterogeneity in variances. For proportion or percentage data, this is not advocated as a good general choice. The arcsine square-root transformation or the folded-exponential family of transformations (24) could be considered but we found the square-root transformation on our data set to be the most appropriate (data not shown). Flowering time was treated as a covariate because it has been known to explain variability in adult resistance (32). The flowering time data were centered and scaled to have a mean of zero and variance of one prior to analysis. This allows for a more meaningful interpretation of the intercept, which represents the expected value of the response when the covariate equals zero. Unless these data are centered, the intercept would correspond to an arbitrary value that falls outside the range of the covariate data (29).

Diseased leaf area data were examined by both multivariate and univariate modeling techniques. Data analysis for each modeling technique was conducted in two stages. The data from single environments were analyzed first, followed by a multi-environment analysis of all of the data collected, considering locationyear combinations as a set of four distinct environments.

The following multivariate MLM was used for single-environment analyses in accordance with the notation used by Apiolaza et al. (3):

$$
Y_{i j k l}=X_{i j k l} \beta+Z_{\mathrm{R} i} r+Z_{\mathrm{B}(\mathrm{R}) i j} b+Z_{\mathrm{G}(\mathrm{C}) k l} g+\varepsilon
$$

where $Y_{i j k l}=\left[y_{i j k l, 1}, y_{i j k l, 2}, y_{i j k l, 3}, y_{i j k l, 4}\right]^{\mathrm{T}}$ is the vector of ratings at times $t=1,2,3$, and 4 ; and $\beta=\left[\beta^{\mathrm{T}}, \beta^{\mathrm{T}}{ }_{2}, \beta^{\mathrm{T}}{ }_{3}, \beta^{\mathrm{T}}{ }_{4}\right]^{\mathrm{T}}$ is the vector of fixed effects, where each $\beta^{\mathrm{T}}$ is a vector containing (at time $t$ ) the mean, the effect of flowering time, and the effect of a qualitatively distinguishable lesion type (for $\mathrm{NC07}$ and $\mathrm{NC0} 8$ only). Likewise, the vectors $r=\left[r^{\mathrm{T}}, r^{\mathrm{T}}, r^{\mathrm{T}}, r^{\mathrm{T}}\right]^{\mathrm{T}}, b=\left[b^{\mathrm{T}}{ }_{1}, b^{\mathrm{T}}{ }_{2}\right.$, $\left.b^{\mathrm{T}}{ }_{3}, b^{\mathrm{T}}{ }_{4}\right]^{\mathrm{T}}$, and $g=\left[g^{\mathrm{T}}{ }_{1}, g^{\mathrm{T}}{ }_{2}, g^{\mathrm{T}}{ }_{3}, g^{\mathrm{T}}{ }_{4}\right]^{\mathrm{T}}$ contain the time-specific random effects of replications, blocks nested within replications and genotypes nested within cycles. $X_{i j k l}$ is the fixed-effect design matrix relating $\beta$ to $Y_{i j k l}$; and $Z_{\mathrm{R} i}, Z_{\mathrm{B}(\mathrm{R}) i j}$, and $Z_{\mathrm{G}(\mathrm{C}) k l}$ are randomeffect design matrices relating the $i$ th replication, $j$ th block nested within the $i$ th replication, and $l$ th genotype nested within the $k$ th cycle, respectively, to $Y_{i j k l}$. Finally $\varepsilon_{i j k l}=\left[\varepsilon_{1}, \varepsilon_{2}, \varepsilon_{3}, \varepsilon_{4}\right]^{\mathrm{T}}$ represents the vector of random residuals. The random effect terms and residual follow a multivariate normal distribution (Appendix).

The following multivariate MLM was used for multi-environment analysis:

$$
\begin{gathered}
Y_{i j k l m}=X_{i j k l m} \beta+Z_{\mathrm{E} m} e+Z_{\mathrm{R}(\mathrm{E}) m i} r+Z_{\mathrm{B}(\mathrm{R}(\mathrm{E})) m i j} b+Z_{\mathrm{G}(\mathrm{C}) k l} g+ \\
Z_{\mathrm{G}(\mathrm{C}) \mathrm{E} k l m} g e+\varepsilon_{i j k l m}
\end{gathered}
$$

where model 1 was expanded to include $Z_{\mathrm{E} m} e$ and $Z_{\mathrm{G}(\mathrm{C}) \mathrm{Eklm}} g e$. The vectors $e=\left[e^{\mathrm{T}}, e_{1}^{\mathrm{T}}, e^{\mathrm{T}}{ }_{3}, e^{\mathrm{T}}{ }_{4}\right]^{\mathrm{T}}$ and ge $=\left[g e^{\mathrm{T}}, g e^{\mathrm{T}}{ }_{2}, g e^{\mathrm{T}}{ }_{3}, g e^{\mathrm{T}}\right]^{\mathrm{T}}$ contain the time-specific random effects of environments and genotype-environment $(\mathrm{G} \times \mathrm{E})$ interactions. The design matrices $Z_{\mathrm{E} m}$ and $Z_{\mathrm{G}(\mathrm{C}) \mathrm{E} k l m}$ relate the random effects of the $m$ th environment and the $l$ th $-m$ th $\mathrm{G} \times \mathrm{E}$ interaction, respectively to $Y_{i j k l m}$. Also, for the multi-environment analysis, replications and blocks nested within replications were modeled as nested within environments. The random effect terms and residual again follow a multivariate normal distribution (Appendix).

Single-environment analyses were conducted first to examine patterns in variation across time in each of the four environments. The following covariance and correlation structures were fit to model the across time variation in the random effect terms and residual and their fit was evaluated using the Akaike Information Criterion (1). A brief description of the matrix structures is given here but further details can be found in Apiolaza and Garrick (2): (i) US is an unstructured covariance matrix with $n \times(n+1) / 2$ parameters to be estimated, where $n=$ the number of ratings; (ii) CORUH is a uniform correlation matrix with heterogeneous/ unequal variances at each time and $n+1$ parameters to be estimated; (iii) AR1H is a first-order autoregressive correlation matrix with $n+1$ parameters to be estimated (i.e., exponentially declining correlations across time, with heterogeneous or unequal variances at each time; (iv) $\mathrm{CORBH}$ is a banded correlation matrix with $2 n-1$ parameters to be estimated (i.e., specific correlations for each time lag, with heterogeneous or unequal variances at each time); and (v) homogeneous variance versions of the above have $n-1$ fewer parameters to be estimated. The best-fitting covariance structures obtained from single-environment analyses were used to guide the development of the multi-environment model.

As a cautionary note for multivariate analysis in ASReml, the terms "factor" (corresponding to a random effect factor fit across time) and "Trait.factor" (corresponding to the same random effect factor fit at each time) should not be modeled simultaneously when any covariance structure other than the case when zero correlation and homogeneous variance is modeled on Trait. This is a mis-specified model that will lead to singularities or negative variance components in the estimated covariance matrix.

Variances estimated in single and multi-environment analyses were used to calculate time-specific narrow-sense heritabilities $\left(h^{2}\right)$ on a family-mean basis, using time-specific harmonic means in the formula given by Holland et al. (13).

For univariate analysis, AUDPC was calculated as follows:

$$
\text { AUDPC }=\left[\sum_{t=1}^{r-1}\left(\left(\frac{y_{t}+y_{t+1}}{2}\right) \times\left(\mathrm{d}_{t+1}-\mathrm{d}_{t}\right)\right)\right] \div\left(\mathrm{d}_{r}-\mathrm{d}_{0}\right)
$$

where $y_{t}$ was the observed data at time $t$ and $d_{t}$ was the $t$ th day of measurement, with $t=1$ to 4 measurements ( $r=4$ was the total number of observations). Standardization by the divisor $\left(\mathrm{d}_{r}-\mathrm{d}_{0}\right)$ scaled AUDPC to the original 0 to $100 \%$ scoring scale.

The following univariate MLM was used for single-environment analyses of AUDPC:

$$
y_{i j k l}=\mu+f_{i j k l}+b_{i j k l}+\mathrm{R}_{i}+\mathrm{B}(\mathrm{R})_{i j}+\mathrm{G}(\mathrm{C})_{k l}+\varepsilon_{i j k l}
$$

where $y_{i j k l}$ is the square-root transformation of AUDPC, $\mu$ is the overall mean, $f_{i j k l}$ is the fixed effect of flowering time, $b_{i j k l}$ is the fixed effect of a qualitatively distinguishable lesion type (for $\mathrm{NC07}$ and NC08 only), $\mathrm{R}_{i}$ is the random effect of the $i$ th replication, $\mathrm{B}(\mathrm{R})_{i j}$ is the random effect of the $j$ th incomplete block nested within the $i$ th replication, $\mathrm{G}(\mathrm{C})_{k l}$ is the random effect of the $l$ th genotype nested within the $k$ th cycle, and $\varepsilon_{i j k l}$ is the random error (residual). The random effect terms and residual are all 
assumed to be sampled from normal distributions with means of zero and variances of $\sigma_{\mathrm{R}}^{2}, \sigma_{\mathrm{B}(\mathrm{R})}^{2}, \sigma_{\mathrm{G}(\mathrm{C})}^{2}$, and $\sigma^{2}$, respectively.

The following univariate MLM was used for multi-environment analysis of AUDPC:

$$
\begin{gathered}
y_{i j k l m}=\mu+f_{i j k l m}+b_{i j k l m}+\mathrm{E}_{m}+\mathrm{R}(\mathrm{E})_{m i}+\mathrm{B}(\mathrm{R}(\mathrm{E}))_{m i j} \\
+\mathrm{G}(\mathrm{C})_{k l}+\mathrm{G}(\mathrm{C}) \mathrm{E}_{k l m}+\varepsilon_{i j k l m}
\end{gathered}
$$

Similar to the multivariate analysis for the multi-environment model 2, the terms $\mathrm{E}_{m}$ and $\mathrm{G}(\mathrm{C}) \mathrm{E}_{k l m}$ were added to model 4 as random effects corresponding to the $m$ th environment and the $l$ th$m$ th $\mathrm{G} \times \mathrm{E}$ interaction, respectively. Also, replications and blocks nested within replications were modeled as nested within environments. Here, the random effect terms and residual are all assumed to be sampled from independent normal distributions with means of zero and variances $\sigma_{\mathrm{E}}^{2}, \sigma_{\mathrm{R}(\mathrm{E})}^{2}, \sigma_{\mathrm{B}(\mathrm{R}(\mathrm{E}))}^{2}, \sigma_{\mathrm{G}(\mathrm{C}) \mathrm{E}}^{2}$, and $\sigma^{2}$, respectively. Similar to the procedure followed for multivariate analysis, $h^{2}$ was calculated on a family-mean basis for singleenvironment and multi-environment analyses.

Empirical best linear unbiased estimates (EBLUEs) and empirical best linear unbiased predictors (EBLUPs) were estimated from each model. For both multivariate and univariate analyses, in order to make inferences on the original rating scale, we backtransformed (squared) the sum of the corresponding time-specific intercepts (EBLUEs) and time-specific genotype estimates (EBLUPs) for further analysis. This was done to present the results on their original scale. Back-transformations of the estimates from data initially transformed via a square root transformation are equal to medians instead of means. Considering the initial nonsymmetric distribution observed for our NLB raw data, only medians of that distribution are equivalent to means of the transformed (symmetric) distribution used to arrive at estimates produced from statistical modeling; thus, inferences made on the original scale after back-transformation are to be interpreted in terms of estimated medians and not means.

Model fitting was attempted with ASReml-2 and SAS 9.2 software. We found ASReml-2 to be more computationally efficient when fitting complex covariance structures in multivariate MLMs. Whereas ASReml completed running a single-environment model in less than half a minute, SAS took $>7 \mathrm{~h}$ to run the same model. All models were fit using ASReml-2 (single-environment analysis) and ASreml-Discovery (this version became available during manuscript revisions in which the multi-environment analysis was redone) by restricted maximum likelihood $(18,22)$. Graphics were produced using R.

Simulation analysis. A computer simulation was programmed in $\mathrm{R}$ based on the results of the multivariate analysis of NLB (Table 1) to examine the impact of the number and timing of disease ratings on $h^{2}$ (an index-based $h^{2}$ was calculated from covariance matrices following the method of Kump et al. [15]). The simulation analysis required covariance or correlation matrices for genotype, $\mathrm{G} \times \mathrm{E}$, and residual, containing values at time points for which we had no real data. For the genotype and $\mathrm{G} \times \mathrm{E}$ terms, variance interpolation was achieved by fitting a cubic spline function (R stats package: "spline") between time-specific variances obtained from our model (Table 1) and time. The correlations between time points were modeled under an autoregressive pattern of decay across time. Different scenarios were examined in which the genotypic correlation between consecutive days was assumed to be $1.000,0.995,0.990,0.950$, or 0.990 . Thus, the genotypic correlation between the first and last day of rating across the 27-day time frame simulated (mimicking our data set) was $1.000,0.873,0.762,0.250$, or 0.058 (e.g., $0.990^{27}=$ 0.058 ), respectively. For each of these scenarios, the $\mathrm{G} \times \mathrm{E}$ correlation between consecutive days was assumed to be the product of that estimated from the real data set (i.e., 0.971) and the assumed

\begin{tabular}{|c|c|c|c|c|c|c|c|}
\hline \multirow[b]{2}{*}{ Factor $^{\mathrm{a}}$} & \multirow[b]{2}{*}{ Univariate $^{\mathrm{b}}$} & \multicolumn{4}{|c|}{ Longitudinalc $^{c}$} & \multirow[b]{2}{*}{ Time } & \multirow[b]{2}{*}{$\operatorname{Cov}^{\mathrm{d}}$} \\
\hline & & 1 & 2 & 3 & 4 & & \\
\hline Incomplete block & 0.026 & $\begin{array}{l}\mathbf{0 . 0 1 1} \\
0.012 \\
0.012 \\
0.009\end{array}$ & $\begin{array}{l}0.733 \\
\mathbf{0 . 0 2 7} \\
0.025 \\
0.019\end{array}$ & $\begin{array}{l}0.538 \\
0.733 \\
\mathbf{0 . 0 4 4} \\
0.033\end{array}$ & $\begin{array}{l}0.395 \\
0.538 \\
0.733 \\
\mathbf{0 . 0 4 8}\end{array}$ & $\begin{array}{l}1 \\
2 \\
3 \\
4\end{array}$ & AR1H \\
\hline Replication & 0.078 & $\begin{array}{l}\mathbf{0 . 0 1 3} \\
0.007 \\
0.003 \\
0.002\end{array}$ & $\begin{array}{l}0.512 \\
\mathbf{0 . 0 1 3} \\
0.007 \\
0.004\end{array}$ & $\begin{array}{l}0.262 \\
0.512 \\
\mathbf{0 . 0 1 3} \\
0.007\end{array}$ & $\begin{array}{l}0.134 \\
0.262 \\
0.512 \\
\mathbf{0 . 0 1 3}\end{array}$ & $\begin{array}{l}1 \\
2 \\
3 \\
4\end{array}$ & AR1V \\
\hline Environment (E) & 0.830 & $\begin{array}{l}\mathbf{1 . 1 8 8} \\
1.108 \\
1.034 \\
0.965\end{array}$ & $\begin{array}{l}0.933 \\
\mathbf{1 . 1 8 8} \\
1.129 \\
1.072\end{array}$ & $\begin{array}{l}0.870 \\
0.933 \\
\mathbf{1 . 1 8 8} \\
1.129\end{array}$ & $\begin{array}{l}0.812 \\
0.870 \\
0.933 \\
\mathbf{1 . 1 8 8}\end{array}$ & $\begin{array}{l}1 \\
2 \\
3 \\
4\end{array}$ & AR1V \\
\hline Genotype (G) & 0.313 & $\begin{array}{l}\mathbf{0 . 1 0 9} \\
0.170 \\
0.217 \\
0.207\end{array}$ & $\begin{array}{l}0.991 \\
\mathbf{0 . 2 6 8} \\
0.342 \\
0.324\end{array}$ & $\begin{array}{l}0.982 \\
0.991 \\
\mathbf{0 . 4 4 8} \\
0.424\end{array}$ & $\begin{array}{l}0.972 \\
0.982 \\
0.991 \\
\mathbf{0 . 4 1 3}\end{array}$ & $\begin{array}{l}1 \\
2 \\
3 \\
4\end{array}$ & AR1H \\
\hline $\mathrm{G} \times \mathrm{E}$ & 0.047 & $\begin{array}{l}\mathbf{0 . 0 4 6} \\
0.036 \\
0.027 \\
0.021\end{array}$ & $\begin{array}{l}0.770 \\
\mathbf{0 . 0 4 6} \\
0.045 \\
0.045\end{array}$ & $\begin{array}{l}0.593 \\
0.770 \\
\mathbf{0 . 0 4 6} \\
0.045\end{array}$ & $\begin{array}{l}0.456 \\
0.593 \\
0.770 \\
\mathbf{0 . 0 4 6}\end{array}$ & $\begin{array}{l}1 \\
2 \\
3 \\
4\end{array}$ & AR1V \\
\hline Residual & 0.195 & $\begin{array}{l}\mathbf{0 . 1 2 7} \\
0.092 \\
0.080 \\
0.066\end{array}$ & $\begin{array}{l}0.487 \\
\mathbf{0 . 2 8 1} \\
0.185 \\
0.136\end{array}$ & $\begin{array}{l}0.360 \\
0.561 \\
\mathbf{0 . 3 8 8} \\
0.236\end{array}$ & $\begin{array}{l}0.309 \\
0.429 \\
0.633 \\
\mathbf{0 . 3 5 9}\end{array}$ & $\begin{array}{l}1 \\
2 \\
3 \\
4\end{array}$ & US \\
\hline
\end{tabular}

TABLE 1. Covariance estimates from univariate and multivariate analysis of the multi-environment data set

${ }^{\text {a }}$ Each of the factors was significant at $P<0.001$ according to the likelihood ratio test.

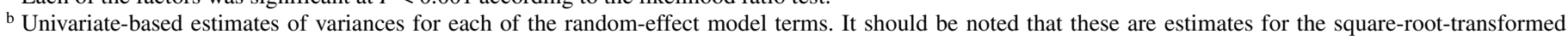
model.


(above the bold diagonal) for each of the random-effect model terms. These too are estimates for the square-root transformed model.

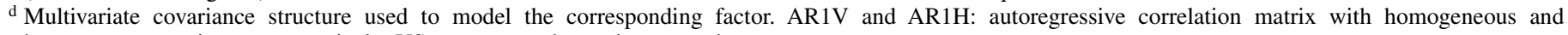
heterogeneous variances, respectively; US: unstructured covariance matrix. 
genotypic correlation from above (e.g., 1.00, 0.995, and so on). This maintained the ratio of genotypic to $\mathrm{G} \times \mathrm{E}$ correlation across the levels of genotypic correlation modeled.

The residual correlation was unstructured, providing no way of interpolating pairwise correlations for simulated days or unobserved information. In each of 100,000 replications of simulation, an unstructured correlation matrix was created with pairwise correlations ( $r$ ) obtained from the inverse Fisher's $r$ to $z$ transformation; $z$ values were drawn from a standard normal distribution.

The simulation was used to examine two different temporal rating scenarios: (i) ratings taken approximately evenly across time of the entire rating period from day 1 to 28 and (ii) ratings taken daily in a constrained time-window surrounding the day at which $h^{2}$ was minimum in one scenario and maximum in another (for the NLB data set, $h^{2}$ was lowest at 1 day and greatest at 20 days from the first rating for the multi-environment analysis) (Fig. 1). In each scenario, the number of ratings examined was 2 , 6 , and 10 .

Simulations led to the creation of covariance matrices specific to each different scenario. Positive-definiteness of the matrices was ensured by using the make.positive.definite function in $\mathrm{R}$ with a tolerance limit of $10^{-6}$. Finally, the average of the indexbased heritabilities (i.e., those calculated from all of the replications across which the residual covariances varied by sampling) was calculated.

\section{RESULTS AND DISCUSSION}

Accounting for an unexpected covariate: a qualitative lesion phenotype. The inbred founders and the population formed from them that underwent recurrent selection were reported to not contain race-specific resistance genes for NLB, and selection was said to be for quantitative and not qualitative resistance (5). However, in our field trials, we observed a qualitatively different kind of lesion in North Carolina alone, where a mixture of race 1 , race 2,3 , and race 2,3, $\mathrm{N}$ isolates were used for inoculation, which resembled an incompatible race-specific resistance interaction. The lesion type was observed at an appreciable frequency on plants sampled from $\mathrm{C}_{0}(36 \%$ in at least two reps across both years) but was not observed on plants sampled from other cycles or the inbred founders or hybrids between them. A preliminary screening of the metapopulation with molecular markers confirmed that it did, indeed, contain non-founder alleles, which were detected in all cycles (data not shown). This suggested that the population had received alleles from an unknown source prior to the formation of $\mathrm{C}_{0}$ and that there was selection against the qualitatively different type of lesion in the first cycle of selection. In our trials in New York, where only race 1 of S. turcica was used for inoculation, these same types of lesions were never observed; instead, only the water-soaked, wilted lesions typical of quantitative resistance to NLB were observed. $S$. turcica race 1 is virulent on maize genotypes carrying the race-specific resistance gene Htl. Taken together, we inferred that the Htl gene was present in $\mathrm{C}_{0}$ and was either at a low frequency or absent in all later cycles. To control for the large effect of Htl on quantitative resistance in North Carolina trials, it was included as a fixedeffect in the statistical models. The lesion type had a highly significant effect $(P<0.001)$ on disease resistance in both multivariate and univariate analyses.

Multivariate MLM. In both the single- and multi-environment analyses, all of the model terms were significant at $\alpha=0.05$. In the multi-environment analysis, although the effect of genotypes

\section{Genotype}

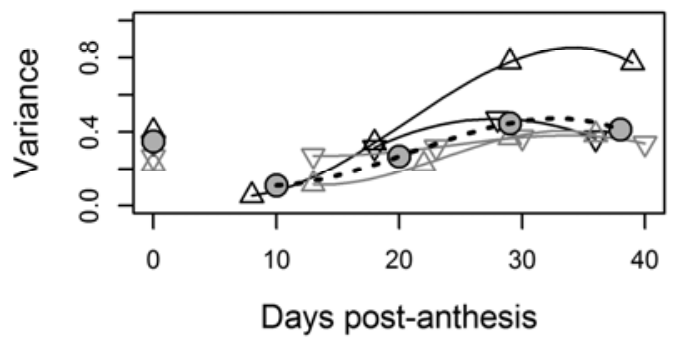

Residual

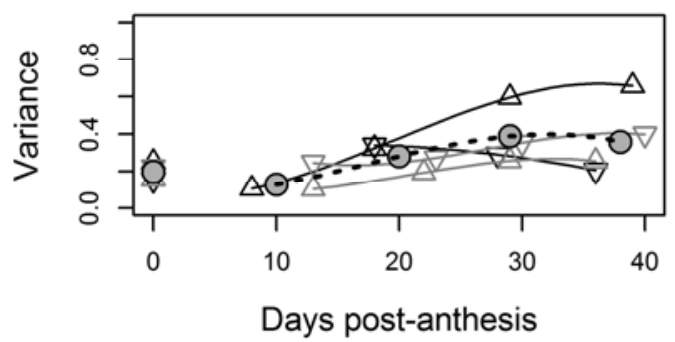

Genotype X Environment

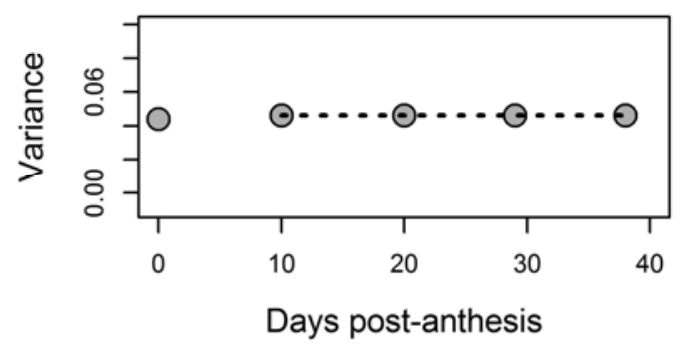

Heritability

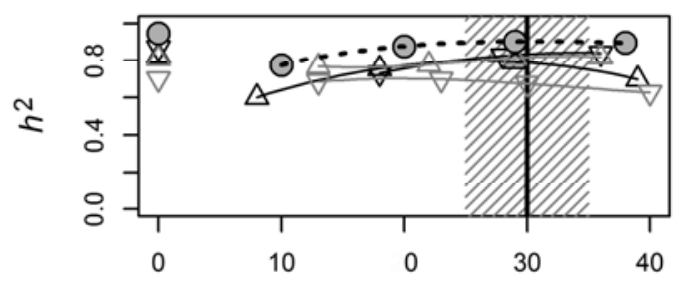

Days post-anthesis

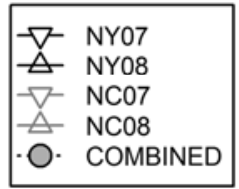

Fig. 1. Estimates of genotype, genotype-environment $(\mathrm{G} \times \mathrm{E})$, and residual variances and $h^{2}$ of northern leaf blight (NLB) diseased leaf area. Multivariate-based estimates of variances and $h^{2}$ are shown as a function of the number of days after the metapopulation average of anthesis, which is often the basis for deciding when NLB disease ratings are to commence. Note that the $\mathrm{y}$-axis scale is different in the G×E panel. A third-degree polynomial function was used to calculate the fitted lines shown in each plot. The vertical line in the $h^{2}$ plot indicates the post-anthesis day at which $h^{2}$ was maximum, with shading at \pm 5 days. The symbols at $\mathrm{x}=0$ in each plot refer to $h^{2}$ estimates from single environment and multi-environment analyses of area under the disease progress curve. 
captured 2.3 to 9.7 times more of the variation than that of $\mathrm{G} \times \mathrm{E}$ (Table 1; Fig. 1), disease development varied among the genotypes across environments. Rank changes among genotypes across environments were evident based on correlations of the G×E EBLUPs (averaged across ratings) between each pair of environments $\left(r_{\text {Spearman }}=0.60\right.$ to 0.77$)$. Therefore, single- and multi-environment analyses were performed throughout this study (it is noteworthy to point out here that time-specific modeling of the $\mathrm{G} \times \mathrm{E}$ correlation [i.e., correlation of genotypes across environments] is also possible in the multivariate MLM, although this was not the focus of our study).

A primary advantage to using multivariate MLM on longitudinal data is that the correlative structure of the repeatedmeasures data across time can be identified and its parameters estimated. For single-environment analysis, the most parsimonious correlative time-dependent structure was AR1H fit to the genotypes, environment-specific combinations of AR1H and AR1V fit to the experimental design factors, and US fit to the residuals. A similar structure, dominated by the AR1 pattern of correlation decay across ratings, was observed for the multienvironment analysis (Table 1).

Based on the multi-environment analysis, the genotypic correlation between NLB ratings separated by $\approx 10$ days was very high $(\hat{p}=0.990)$ (Table 1$)$. This corresponded to a correlation for ratings separated by 1 day of 0.999 and ratings separated by 27 days (i.e., the maximum separation in rating times in our study) of 0.970 , which indicated that disease development occurred similarly among genotypes across time. The high genotypic temporal correlation in the NLB data set led to the expectation of finding few crossover events in the disease profiles of different genotypes. Accordingly, the multivariate analysis revealed subtle differences in the effect of the genotypes on disease development, such that all of the genotypes appeared to follow a similar disease progress curve (Figs. 2A and 3); changes in resistance ranks among genotypes across time were negligible $\left(r_{\text {Spearman }}=0.99\right)$.

As is typical of plant disease development curves, the rate of NLB disease development differed among resistant and susceptible genotypes at different time intervals (Fig. 2). Consequently, a change in the genotypic variance across time was observed (Table 1; Fig. 1), which indicated that the timing of rating could be important for differentiating the effects of treatments on disease development. Heritability provides an estimate of the amount of phenotypic variance attributable to genotypic variation, and statistical power to differentiate genotypes increases with increasing heritability. The $h^{2}$ of NLB resistance peaked at 20 days post-anthesis ( 7 days after the first rating) in $\mathrm{NC} 07,33$ days post-anthesis (21 days after the first rating) in NC08, 34 days post-anthesis (16 days after the first rating) in NY07, 26 days post-anthesis
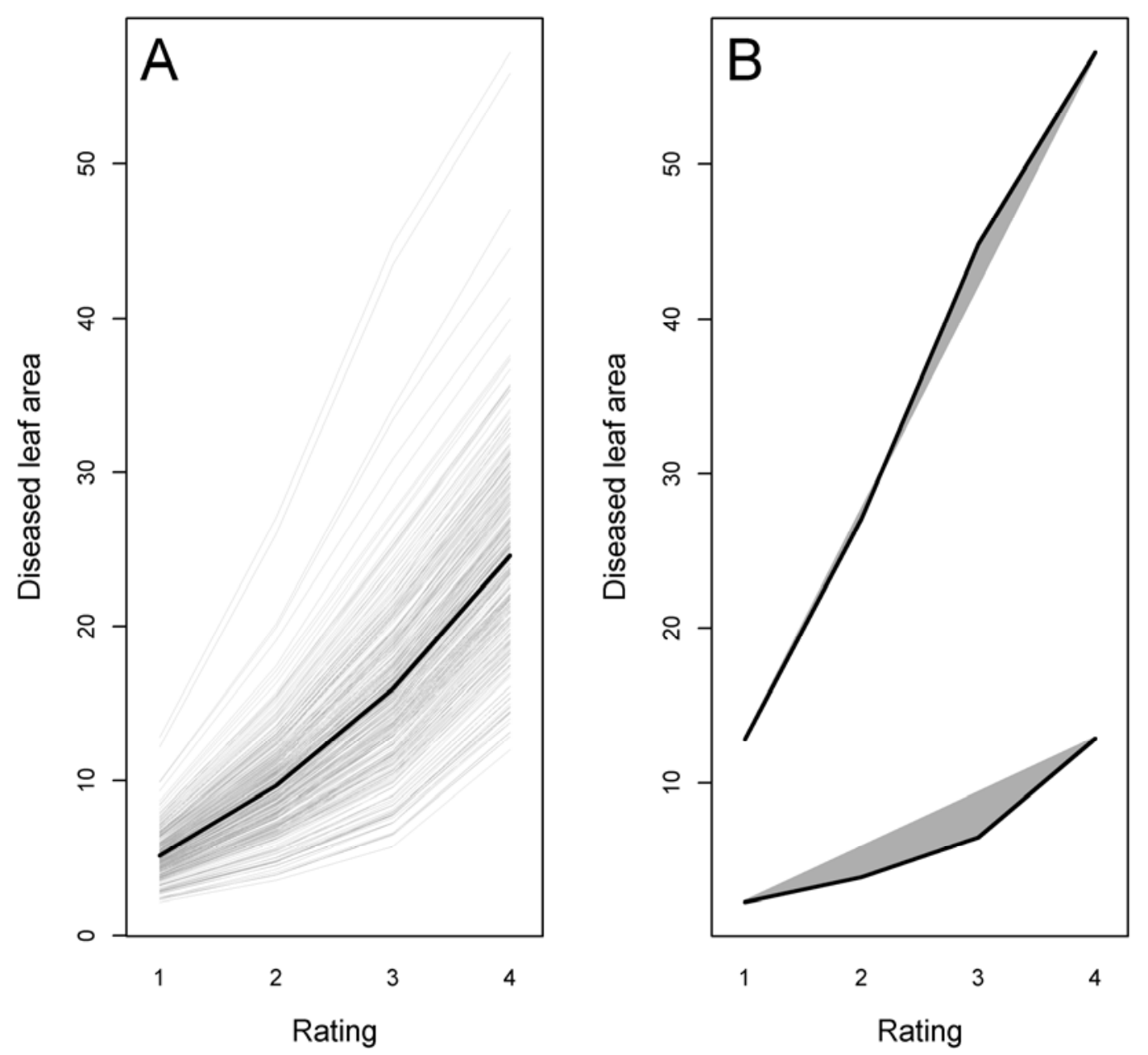

Fig. 2. A, Spaghetti plot of genotype empirical best linear unbiased predictors (EBLUPs) from multivariate mixed linear model analysis of the multi-environment trial. The plot shows the disease development for each genotype based on the back-transformed EBLUPs (lines are connected between rating-specific EBLUPs). The regression line of back-transformed EBLUPs on consecutive rating intervals is shown as a thick black line. B, Representation of the source of bias in genotypic means and variances associated with area under the disease progress curve (AUDPC) calculation when ratings are taken near the beginning and end (in our case, when the plants begin to undergo natural senescence) of an epidemic. Spaghetti plots are shown for the most susceptible and most resistant genotypes (i.e., extremes from A). Shaded portions represent the extent of under- or over-estimation of areas when using the two distal time points compared with four. For the susceptible genotype, the two-point AUDPC is less than the four-point AUDPC. For the resistant genotype, the two-point AUDPC is greater than the four-point AUDPC. Collectively, this is expected to lead to an overall reduction in mean differences between genotypes and variance among genotypes. 

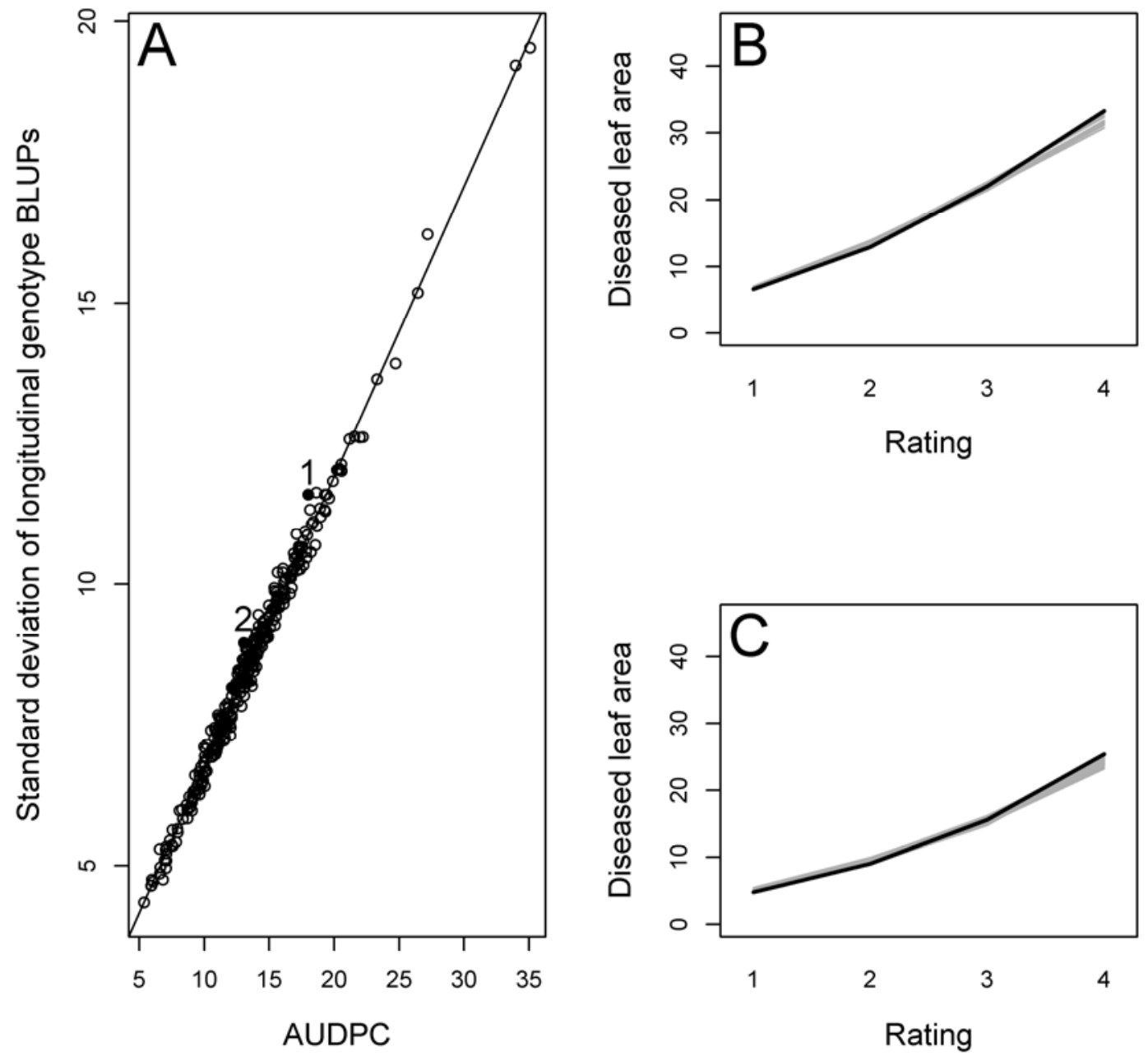

Fig. 3. A, Relationship between the standard deviation among rating-specific empirical best linear unbiased predictors (EBLUPs) (y-axis) and area under the disease progress curve (AUDPC) (x-axis) for each genotype. Both the standard deviations and AUDPCs were calculated from the back-transformed EBLUPs estimated from the multivariate mixed linear model fit to the multi-environment data set. Two genotypes (labeled 1 and 2) were $\geq 3$ standard deviations from the mean of the fitted (black) line and, thus, were identified as extreme valued genotypes. B and C, Differences in disease progress curves for genotypes with AUDPCs similar to those of genotypes 1 and 2 from A. Genotypes $\mathbf{B}, 1$ and $\mathbf{C}, 2$ (shown as black lines) exhibit a more rapid, though subtle, increase in disease development than the genotypes with similar AUDPCs (i.e., genotypes with an AUDPC estimate of 0.5 less than or greater than the "outlier"; these genotypes are shown as gray lines).

(18 days after the first rating) in NY08, and 30 days post-anthesis (20 days after the first rating) in the multi-environment analysis (Fig. 1). If the timing of rating takes place near the point at which $h^{2}$ is at its maximum, regardless of the correlations in the data, by definition, larger proportions of the phenotypic variation can be attributed to genotypic effects. Inferring from the disease epidemics and treatments specific to this study, this suggests that, to collect data near the time at which $h^{2}$ of NLB resistance is maximum, at least one rating should be taken at $\approx 22$ to 32 days post-anthesis or perhaps soon before natural senescence begins to reduce the variation in disease-related senescence. We do not advocate this as a general recommendation for disease scoring but, instead, use this to demonstrate that there is a point in time when $h^{2}$ may be maximal for a given experimental system, which can be identified via multivariate MLM analysis and aid in temporal inference.

Univariate MLM. On grounds of observing high genotypic correlations between ratings in the multivariate analysis, univariate analysis of AUDPC was expected to lead to similar to those conclusions reached based on the multivariate analysis. Indeed, the univariate MLM test results from both single- and multienvironment environment analyses corroborated those obtained from the multivariate analyses. First, significant effects were detected for all of the model terms. Second, the multi-environment analysis of AUDPC revealed highly significant genotype and G $\times E$ effects (Table 1), with genotypic effects consistently explaining more of the variation than $\mathrm{G} \times \mathrm{E}$ in all analyses. Finally, the AUDPC-based $h^{2}$ as obtained from the multi-environment analysis $(0.913)$ was practically identical to the index-based $h^{2}(0.922)$ calculated from the results of multivariate analysis.

Based on these results, AUDPC was determined to be a suitable measure for inference on genotypic performance in our trials. This conclusion was scrutinized using output from the multivariate analysis. The potential drawback of using AUDPC is that genotypes differing in disease response profiles could have similar AUDPCs (27). Using the estimated effects from multivariate analysis, we devised an exploratory approach to identify genotypes with substantially varying response profiles (i.e., crossovers in resistance levels across time) by plotting the standard deviation of time-specific EBLUPs against AUDPC calculated from timespecific EBLUPs of all genotypes (Fig. 3). Although we expected this procedure to allow for the identification of even rare, unique profiles among the many treatment levels, none of the genotypes in our data set exhibited substantially different disease profiles. Two genotypes were identified for which the estimated disease development trend exhibited relatively high deviation (i.e., $\geq 3$ standard deviation from the regression line) (Fig. 3) but the response profiles of these genotypes were not meaningfully dif- 
TABLE 2. Heritabilities based on different numbers and timing of disease rating from data with different levels of temporal correlation among genotypes

\begin{tabular}{|c|c|c|c|c|c|c|c|c|c|c|}
\hline \multirow[b]{3}{*}{ Corg-1int ${ }^{\mathrm{a}}$} & \multirow[b]{3}{*}{ Corg-27int ${ }^{b}$} & \multicolumn{9}{|c|}{ Number of ratings ${ }^{c}$} \\
\hline & & \multicolumn{3}{|c|}{2} & \multicolumn{3}{|c|}{6} & \multicolumn{3}{|c|}{10} \\
\hline & & $\operatorname{Min} h^{2}$ & Equal & $\operatorname{Max} h^{2}$ & $\operatorname{Min} h^{2}$ & Equal & $\operatorname{Max} h^{2}$ & $\operatorname{Min} h^{2}$ & Equal & $\operatorname{Max} h^{2}$ \\
\hline 1.000 & 1.000 & 0.86 & 0.92 & 0.94 & 0.91 & 0.96 & 0.96 & 0.93 & 0.96 & 0.96 \\
\hline 0.998 & 0.947 & 0.86 & 0.92 & 0.94 & 0.91 & 0.96 & 0.96 & 0.93 & 0.96 & 0.96 \\
\hline 0.996 & 0.897 & 0.86 & 0.92 & 0.94 & 0.91 & 0.96 & 0.96 & 0.93 & 0.96 & 0.96 \\
\hline 0.994 & 0.850 & 0.86 & 0.92 & 0.94 & 0.91 & 0.96 & 0.96 & 0.93 & 0.96 & 0.96 \\
\hline 0.992 & 0.805 & 0.86 & 0.92 & 0.94 & 0.91 & 0.96 & 0.96 & 0.93 & 0.96 & 0.96 \\
\hline 0.990 & 0.762 & 0.86 & 0.92 & 0.94 & 0.91 & 0.96 & 0.96 & 0.93 & 0.96 & 0.96 \\
\hline 0.950 & 0.250 & 0.86 & 0.90 & 0.94 & 0.91 & 0.95 & 0.96 & 0.93 & 0.96 & 0.96 \\
\hline 0.900 & 0.058 & 0.86 & 0.89 & 0.94 & 0.90 & 0.94 & 0.96 & 0.93 & 0.95 & 0.96 \\
\hline
\end{tabular}

${ }^{a}$ Correlation among genotypes between ratings separated by a 1-day interval. This is the maximum correlation possible.

${ }^{b}$ Correlation among genotypes between ratings separated by a 27 -day interval. This is the minimum correlation possible.

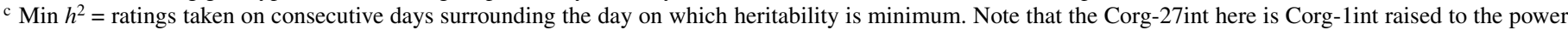
of the number of ratings minus one (i.e., the number of days separating the terminal ratings). Equal = ratings taken at equal intervals across a 1- to 28-day time frame. Max $h^{2}=$ ratings taken on consecutive days surrounding the day on which heritability is maximum. Note that the Corg-27int here is Corg-1int raised to the power of the number of ratings minus one (i.e., the number of days separating the terminal ratings).

ferent from genotypes with similar AUDPCs. For data that are demonstrably less correlated, using AUDPC could lead to improper or limited conclusions (27) and the technique presented here is one way to identify interesting outliers. This is especially useful in situations where differential responses are not readily visible in response profile plots containing numerous treatment levels (e.g., Fig. 2A or studies in which thousands of treatment levels are examined [17,33]).

Simulation analysis on how the number and timing of disease ratings affects estimates of $\boldsymbol{h}^{\mathbf{2}}$. For longitudinal data on plant disease, increasing the number of ratings and having appropriately timed ratings is expected to provide more accurate estimates of response profiles and improved measurement precision (Fig. 2B). In turn, improved precision increases $h^{2}$ and statistical power of hypothesis tests involving the treatments. Observing that $h^{2}$ was not constant across time led us to consider how these different factors influence $h^{2}$, at different levels of correlation among genotypes across time. An index-based measure of $h^{2}$ (15) was used for this analysis, which is a single measure calculated from the average of the covariance matrices; in fact, for data sets in which there are no missing data, the index-based $h^{2}$ corresponds to AUDPC-based $h^{2}$. Here, we distinguish the indexbased from time-specific $h^{2}$ as $h_{\text {index }}^{2}$.

We were initially surprised to see that, even for data with practically no genotypic correlation, relatively high estimates of $h^{2}$ index could be obtained (Table 2). For instance, $h^{2}$ index calculated from two ratings in which the correlation between them was as low as 0.058 equaled no less than 0.86 . This is because the total variation is only partially reduced by reductions in covariation. In the case of our assumed and simulated parameters, the maximum decrease in $h^{2}$ index due to a reduction in covariation alone was 3.20 percentage points (Table 2; c.f. $h^{2}$ estimates from when two ratings were taken and the genotypic correlation equaled $\approx 0.0$ versus 1.0 ).

Regardless of the genotypic correlation assumed, $h^{2}$ index increased with each additional rating (from 2 to 10 ratings) to a maximum of 0.93 to 0.96 (Table 2, c.f. $h^{2}$ in a row-wise fashion for common temporal rating approaches). The largest increases in $h^{2}$ index that could be achieved by taking more ratings occurred when equally spaced ratings were taken and the genotypic correlation across time was very low or when ratings were taken around the time when time-specific $h^{2}$ was the least.

The timing of rating had a clear impact on obtaining the highest estimate of $h^{2}$ possible. Taking as few as two ratings around the time at which time-specific $h^{2}$ was maximum was better than taking 10 ratings around the time at which time-specific $h^{2}$ was minimum. Overall, the simulation results suggested that as few as two ratings are all that are needed to obtain near-maximum estimates of $h^{2}$. If it is possible to take ratings roughly around the peak of the time where $h^{2}$ is likely to peak (if such prior information is available), a single rating would likely suffice.

The simulation analysis considered the distribution of variances across time but not the trade-off between maximizing $h^{2}$ and understanding patterns in disease development. When the genotypic correlation is low across the period of disease development, relative differences among treatment levels at one time would not reflect those at another time. In such an instance, it may be inappropriate to convert longitudinal data to disease progress curves and, instead, be desirable to determine the specific times at which information on resistance is most relevant (e.g., when disease most highly impacts yield). Multivariate analysis provides a way of assessing the correlation between ratings and the relative importance of time-specific disease resistance on yield or other responses of interest.

Summary. The process of conducting multivariate MLM analysis is more involved than univariate MLM analysis and includes the provision to model covariance structures for the random effects in the model and the residual. The combination of a proper understanding of the design and treatment structures (8), data organization (in this case in an ASReml-readable format) (12), correctness of ASReml syntax to facilitate the building of the multivariate MLM framework (12), and suitability of the candidate covariance structures for the data (based on the number of parameters to be estimated, the interpretability of those structures, and diagnostic results) $(2,17)$ will increase the possibility of fitting a suitably parameterized model. To aid in the application of multivariate MLM analysis of longitudinal data on plant disease, the data set modeled in this study and annotated ASReml syntax is provided (Supplementary files 1 and 2).

Multivariate MLM analysis could be beneficial to several applications in plant pathology because it allows multiple scopes of temporal inference to be achieved, which have been highlighted throughout this article. Unlike other approaches to modeling disease development, the approach does not make assumptions about the pattern of disease development ahead of model fitting, and it can be useful on its own or as a pre-modeling approach to understand the structure of plant disease resistance data and to predict missing observations. Although fitting multivariate MLMs may not always uncover interesting biology, which was determined retrospectively to be the case for our NLB data set, the procedure opens up the opportunity for such discovery.

\section{APPENDIX}

Specification of the multivariate MLM. The joint multivariate normal distribution (MND) of $y_{i j k l m}, e, r, b, g$, ge, and $\varepsilon_{i j k l m}$ in model 2 is given by 




where the mean vector comprises the expected values of the observations $\left(y_{i j k l m}\right)$, random effect terms $(e, r, b, g$, and $g e)$, and residual $\left(\varepsilon_{i j k l m}\right)$ and the covariance of the MND comprises covariance matrices for each term $\left(y_{i j k l m}, e, r, b, g, g e\right.$, and $\left.\varepsilon_{i j k l m}\right)$ along the diagonal and covariance matrices between terms in the offdiagonal.

$$
\begin{gathered}
V_{i j k l m}=Z_{\mathrm{E}} \Sigma_{\mathrm{E}} Z^{\mathrm{T}}{ }_{\mathrm{E} m}+Z_{\mathrm{R}(\mathrm{E}) m i} \Sigma_{\mathrm{R}(\mathrm{E})} Z_{\mathrm{R}(\mathrm{E}) m i}^{\mathrm{T}}+ \\
Z_{\mathrm{B}(\mathrm{R}(\mathrm{E})) m i j} \Sigma_{\mathrm{B}(\mathrm{R}(\mathrm{E}))} Z_{\mathrm{B}(\mathrm{R}(\mathrm{E})) m i j}^{\mathrm{T}}+Z_{\mathrm{G}(\mathrm{C}) k l} \Sigma_{\mathrm{G}(\mathrm{C})} Z_{\mathrm{G}(\mathrm{C}) k l}^{\mathrm{T}}+ \\
Z_{\mathrm{G}(\mathrm{C}) \mathrm{E} k l m} \Sigma_{\mathrm{G}(\mathrm{C}) \mathrm{E}} Z_{\mathrm{G}(\mathrm{C}) \mathrm{E} k l m}^{\mathrm{T}}+\Sigma_{\varepsilon}
\end{gathered}
$$

where $V_{i j k l m}$ represents the variance covariance matrix of the observations, and $\Sigma_{\mathrm{E}}, \Sigma_{\mathrm{R}(\mathrm{E})}, \Sigma_{\mathrm{B}(\mathrm{R}(\mathrm{E}))}, \Sigma_{\mathrm{G}(\mathrm{C})}, \Sigma_{\mathrm{G}(\mathrm{C}) \mathrm{E}}$, and $\Sigma_{\varepsilon}$ are the covariance matrices for the random effect terms and the residual (see below), respectively. $Z_{\mathrm{E} m}, Z_{\mathrm{R}(\mathrm{E}) m i}, Z_{\mathrm{B}(\mathrm{R}(\mathrm{E}) m i j}, Z_{\mathrm{G}(\mathrm{C}) k l}$, and $Z_{\mathrm{G}(\mathrm{C}) \mathrm{E} k l m}$ are described for models 1 and 2 in the Theory and Approaches section.

The random effect terms were modeled using an AR1H covariance matrix for genotypes nested within cycles $\left(\Sigma_{\mathrm{G}(\mathrm{C})}\right)$ and blocks nested within replications nested within environments $\left(\Sigma_{\mathrm{B}(\mathrm{R}(\mathrm{E}))}\right)$ and an AR1V covariance matrix for environments $\left(\Sigma_{\mathrm{E}}\right)$, replications nested within environments $\left(\Sigma_{\mathrm{R}(\mathrm{E})}\right)$, and $\mathrm{G} \times \mathrm{E}$ interaction $\left(\Sigma_{\mathrm{G}(\mathrm{C}) \mathrm{E}}\right)$. The autoregressive covariance matrix is as follows:

$$
\left[\begin{array}{cccc}
\sigma_{(t=1)}^{2} & \rho \sigma_{(t=1)} \sigma_{(t=2)} & \rho^{2} \sigma_{(t=1)} \sigma_{(t=3)} & \rho^{3} \sigma_{(t=1)} \sigma_{(t=4)} \\
\rho \sigma_{(t=2)} \sigma_{(t=1)} & \sigma_{(t=2)}^{2} & \rho \sigma_{(t=2)} \sigma_{(t=3)} & \rho^{2} \sigma_{(t=2)} \sigma_{(t=4)} \\
\rho^{2} \sigma_{(t=3)} \sigma_{(t=1)} & \rho \sigma_{(t=3)} \sigma_{(t=2)} & \sigma_{(t=3)}^{2} & \rho \sigma_{(t=3)} \sigma_{(t=4)} \\
\rho^{3} \sigma_{(t=4)} \sigma_{(t=1)} & \rho^{2} \sigma_{(t=4)} \sigma_{(t=2)} & \rho^{3} \sigma_{(t=4)} \sigma_{(t=3)} & \sigma_{(t=4)}^{2}
\end{array}\right]
$$

where the diagonal elements are rating-specific variances (either heterogeneous or homogeneous) and the off-diagonal elements are covariances between ratings. Rho follows a first-order autoregressive pattern of decay.

The residual was modeled using a US matrix $\left(\Sigma_{\varepsilon}\right)$, defined as follows:

$$
\left[\begin{array}{cccc}
\sigma_{(t=1)}^{2} & \sigma_{(t=1,2)} & \sigma_{(t=1,3)} & \sigma_{(t=1,4)} \\
\sigma_{(t=2,1)} & \sigma_{(t=2)}^{2} & \sigma_{(t=2,3)} & \sigma_{(t=2,4)} \\
\sigma_{(t=3,1)} & \sigma_{(t=3,2)} & \sigma_{(t=3)}^{2} & \sigma_{(t=3,4)} \\
\sigma_{(t=4,1)} & \sigma_{(t=4,2)} & \sigma_{(t=4,3)} & \sigma_{(t=4)}^{2}
\end{array}\right]
$$

where the diagonal elements are rating-specific variances and offdiagonal elements are covariances between ratings; each of the elements is individually estimated.

\section{ACKNOWLEDGMENTS}

This research was funded by the United States Department of Agriculture National Institute of Food and Agriculture project 2007-35301$18133 / 19859$. We thank M. Carson for providing seed of the originally selected population and R. Nelson for providing support for field trials conducted in New York.

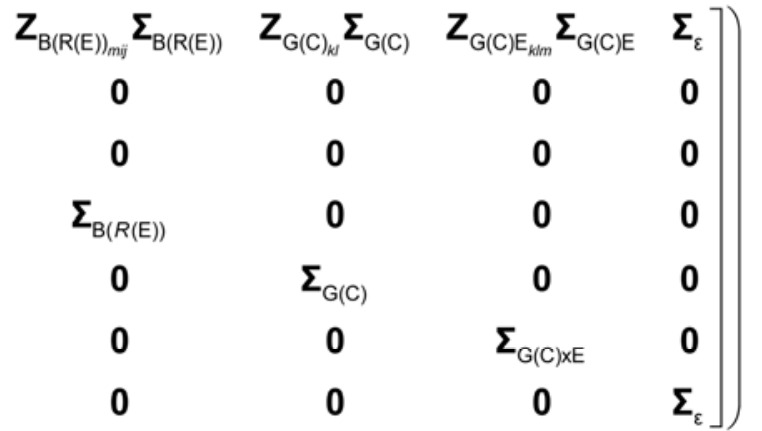

(6)

\section{LITERATURE CITED}

1. Akaike, H. 1974. A new look at the statistical model identification. IEEE Trans. Autom. Control. 19:716-723.

2. Apiolaza, L. A., and Garrick, D. J. 2001. Analysis of longitudinal data from progeny tests: Some multivariate approaches. For. Sci. 47:129-140.

3. Apiolaza, L. A., Gilmour, A. R., and Garrick, D. J. 2000. Variance modelling of longitudinal height data from a Pinus radiata progeny test. Can. J. For. Res. 30:645-654.

4. Campbell, C. L., and Madden, L. V. 1990. Introduction to Plant Disease Epidemiology. John Wiley \& Sons, New York.

5. Carson, M. 2006. Response of a maize synthetic to selection for components of partial resistance to Exserohilum turcicum. Plant Dis. 90:910-914.

6. Contreras-Medina, L., Torres-Pacheco, I., Guevara-González, R., Romero-Troncoso, R., Terol-Villalobos, I., and Osornio-Rios, R. 2009. Mathematical modeling tendencies in plant pathology. Afr. J. Biotechnol. 8:7399-7408.

7. Diggle, P. 2002. Analysis of Longitudinal Data. Oxford University Press, New York.

8. Draper, N. R., and Smith H. 1998. Pages 327-368 in: Applied Regression Analysis. Wiley, New York.

9. Fang, H. 2006. A Monte Carlo study of power analysis of hierarchical linear model and repeated measures approaches to longitudinal data analysis. Ph.D. thesis, Ohio University, Athens.

10. Fitzmaurice, G. M., Laird, N. M., and Ware J. H. 2004. Pages 401-411 in: Applied Longitudinal Analysis. John Wiley \& Sons, New York.

11. Garrett, K., Madden, L., Hughes, G., and Pfender W. 2004. New applications of statistical tools in plant pathology. Phytopathology 94:999-1003.

12. Gilmour A., Gogel B., Cullis B., and Thompson R. 2006. ASReml User Guide Release 2.0. VSN International Ltd., Hemel Hempstead, UK.

13. Holland, J. B., Nyquist, W. E., and Cervantes-Martínez C. T. 2003. Estimating and interpreting heritability for plant breeding: An update. Plant Breed. Rev. 22:9-112.

14. Jeger, M., and Viljanen-Rollinson, S. 2001. The use of the area under the disease-progress curve (AUDPC) to assess quantitative disease resistance in crop cultivars. Theor. Appl. Genet 102:32-40.

15. Kump, K. L., Bradbury, P. J., Wisser, R. J., Buckler, E. S., Belcher, A. R., Oropeza-Rosas, M. A., Zwonitzer, J. C., Kresovich, S., McMullen, M. D., Ware, D., Balint-Kurti, P. J., and Holland, J. B. 2011. Genome-wide association study of quantitative resistance to southern leaf blight in the maize nested association mapping population. Nat. Genet. 43:163-168.

16. Leonard, K. J., and Fry, W. E. 1989. Plant Disease Epidemiology, Vol. 2: Genetics, Resistance, and Management. McGraw-Hill Inc., New York.

17. Littell, R., Henry, P., and Ammerman, C. 1998. Statistical analysis of repeated measures data using SAS procedures. J. Anim. Sci. 76:12161231.

18. Littell, R. C., Milliken, G. A., Stroup, W. W., Wolfinger, R. D., and Schabenberger, O. 2006. SAS for Mixed Models, 2nd ed. SAS Institute Inc., Cary, NC.

19. Madden, L., and Paul, P. 2009. Assessing heterogeneity in the relationship between wheat yield and Fusarium head blight intensity using randomcoefficient mixed models. Phytopathology 99:850-860.

20. Madden, L., and Paul, P. 2010. An assessment of mixed-modeling approaches for characterizing profiles of time-varying response and predictor variables. Phytopathology 100:1015-1029.

21. Madden L. V., Hughes G., and Bosch F. 2007. The Study of Plant Disease Epidemics. The American Phytopathological Society. APS Press, St. Paul, MN.

22. Patterson, H. D., and Thompson, R. 1971. Recovery of inter-block information when block sizes are unequal. Biometrika 58:545-554

23. Paul, P. A., Lipps, P. E., De Wolf, E., Shaner, G., Buechley, G., Adhikari, T., Ali, S., Stein, J., Osborne, L., and Madden, L. V. 2007. A distributed 
lag analysis of the relationship between Gibberella zeae inoculum density on wheat spikes and weather variables. Phytopathology 97:1608-1624.

24. Piepho, H. P. 2003. The folded exponential transformation for proportions. J. R. Stat. Soc. Ser. D. 52:575-589.

25. Poland, J. A., Bradbury, P. J., Buckler, E. S., and Nelson, R. J. 2011. Genome-wide nested association mapping of quantitative resistance to northern leaf blight in maize. Proc. Natl. Acad. Sci. USA 108:6893-6898.

26. Raudenbush, S. W., and Liu, X. 2000. Statistical power and optimal design for multisite randomized trials. Psychol. Methods 5:199-213.

27. Singh, H., and Rao, M. V. 1989. Area under the disease progress curve: its reliability as a measure of slow-rusting resistance. Plant Breed. 103:319323.

28. Van der Plank, J. 1963. Plant Diseases: Epidemics and Control. Academic Press, New York.
29. West, B. T., Welch, K. B., and Galecki, A. T. 2007. Linear Mixed Models: A Practical Guide using Statistical Software. Chapman \& Hall/CRC, Boca Raton, FL.

30. Wisser, R. J., Kolkman, J. M., Patzoldt, M. E., Holland, J. B., Yu J., Krakowsky, M., Nelson, R. J., and Balint-Kurti, P. J. 2011. Multivariate analysis of maize disease resistances suggests a pleiotropic genetic basis and implicates a GST gene. Proc. Natl. Acad. Sci. USA 108:7339-7334.

31. Zadoks, J. C., and Schein, R. D. 1979. Epidemiology and Plant Disease Management. Oxford University Press Inc., New York.

32. Zwonitzer, J. C., Coles, N. D., Krakowsky, M. D., Arellano, C., Holland J. B., McMullen, M. D., Pratt, R. C., and Balint-Kurti, P. J. 2010. Mapping resistance quantitative trait loci for three foliar diseases in a maize recombinant inbred line population-evidence for multiple disease resistance? Phytopathology 100:72-79. 